\title{
Recent minimum-bias measurements by ATLAS
}

\author{
Krzysztof W. Woźniak ${ }^{1, *}$ on behalf of the ATLAS Experiment \\ ${ }^{1}$ Institute of Nuclear Physics, Polish Academy of Sciences Krakow, Poland
}

\begin{abstract}
The Large Hadron Collider provides collisions of protons and nuclei at the highest energies available in the laboratory. A variety of recent results for minimum-bias $p p, p+\mathrm{Pb}$ and $\mathrm{Pb}+\mathrm{Pb}$ collisions obtained by the ATLAS experiment is presented. For $p p$ collisions they include the total and inelastic cross section, properties of minimum-bias events, underlying event characteristics and measurements of diffractive processes. The results for $p+\mathrm{Pb}$ and $\mathrm{Pb}+\mathrm{Pb}$ collisions that are relevant for cosmic ray studies are also presented.
\end{abstract}

\section{Introduction}

The primary goal of the Large Hadron Collider (LHC) is the study of the most energetic collisions of protons in which not only the Standard Model can be verified but also new phenomena may be discovered. The main achievement so far was the discovery of the Higgs particle [1, 2]. Extensive studies of possible new phenomena expected in supersymmetry models and searches for exotic particles, including Dark Matter candidates, were also performed, but no clear evidence of such new physics was seen so far.

In the context of cosmic-ray studies more interesting than such rare processes are results obtained from minimum-bias measurements, which provide an opportunity to test Monte Carlo models used and then to obtain predictions for collision energies exceeding those available in accelerators. The ATLAS detector elements used in these measurements are briefly described in Section 2 . The most basic observable for minimum-bias studies is the $p p$ cross section. Its dependence on the collision energy is presented in Section 3. The global characterisics of particle production in $p p$ events and in the underlying event that accompanies a hard scattering are described in Section 4 , while results on diffractive processes are shown in Section 5. Finally, in Section 6, the dependence of particle production on the collision centrality for $\mathrm{Pb}+\mathrm{Pb}$ and $p+\mathrm{Pb}$ collisions is discussed and most recent results on light-bylight scattering are shortly mentioned.

\section{ATLAS detector}

The ATLAS detector [3] comprises many systems allowing detection and precise measurements of different types of particles produced in the collisions delivered by the LHC. Charged particles are reconstructed in the central pseudorapidity range $|\eta|<2.5$ using information from the Inner Detector (ID) surrounding the interaction point and placed in the 2 Tesla magnetic field. The innermost part

\footnotetext{
*e-mail: krzysztof.wozniak@ifj.edu.pl
}

of ID, the pixel detector, consists of several layers of silicon pixel sensors arranged into a cylindrical barrel and two sets of disks on both sides of the barrel. Similar layouts also have the silicon strip sensors of the Semicondictor Tracker (SCT) placed directly after the pixel detector. Tracks found in these two detectors may be extended to the Transition Radiation Tracker (TRT), which is the outermost part of the ID. The electromagnetic and hadronic calorimeters, placed farther away from the interaction point and covering the range $|\eta|<4.9$, are used to measure the energy of jets and high- $p_{\mathrm{T}}$ particles. Muons with $|\eta|<2.7$, which traverse all the above mentioned detectors without stopping, are registered in the Muon Spectrometers.

In addition to those main systems of the ATLAS detector there are several smaller components providing specific signals needed by the trigger system or used in some special measurements. One of them is the Minimum Bias Trigger Scintillators detector (MBTS) which consists of two disks placed perpendicularly to the beam line at $\pm 3.56 \mathrm{~m}$ from the nominal interaction point. It registers signals from charged particles emitted within the pseudorapidity range $2.08<|\eta|<3.86$. Thanks to the large acceptance of MBTS, it is suitable to trigger, with high efficiency, events used in minimum-bias studies.

Several more small detectors are located at large distances from the interaction point. The ALFA detector [4] using scintillator fibres is installed at $\pm 237 \mathrm{~m}$ and $\pm 241 \mathrm{~m}$. The acceptance range of ALFA is approximately $9.6<$ $|\eta|<12.4$. The ATLAS Forward Proton detector (AFP) [5] is slightly closer, at $\pm 205 \mathrm{~m}$ and $\pm 217 \mathrm{~m}$. Each of its arms has two stations, which comprise 4 planes of silicon pixel sensors and a Time-of-Flight detector. Both ALFA and AFP detectors are placed in Roman Pots very close to the beam and are used to measure particles emitted at very small angles. Closer to the interaction point is the detector of the LHCf experiment [6] located at $\pm 140 \mathrm{~m}$ and measuring photons emitted in the ranges $8.81<|\eta|<8.99$ and $|\eta|>10.96$ using calorimeters. 


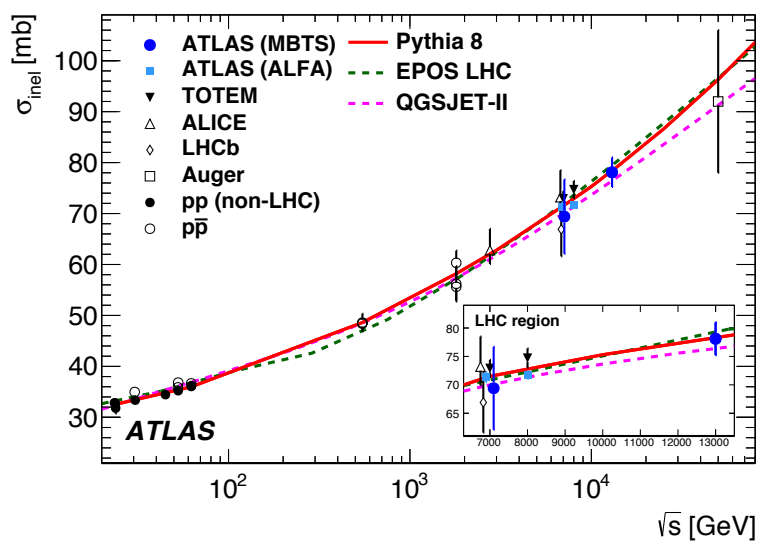

Figure 1. Proton-proton inelastic cross section as a function of collision energy, $\sqrt{s}[9,10]$.

\section{Total and inelastic cross section}

The ALFA detector is placed so far from the interaction point that it can register protons from elastic $p p$ scattering. A coincidence of signals in the two opposite arms of the ALFA detector is a signature of such events. By measuring the elastic cross section as a function of the Mandelstam momentum transfer variable, $t$, and applying the optical theorem it is possible to calculate the total $p p$ cross section. On the other hand the elastic cross section is obtained by integrating the function describing the differential cross section in the full $t$ range. Such measurements were performed for $p p$ collisions at $\sqrt{s}=7 \mathrm{TeV}$ [7] and $\sqrt{s}=8 \mathrm{TeV}$ [8]. The total cross section is $95.35 \pm 0.38$ (stat.) \pm 1.25 (exp.) \pm 0.37 (extr.) mb and $96.07 \pm 0.18$ (stat.) \pm 0.85 (exp.) \pm 0.31 (syst.) mb at $7 \mathrm{TeV}$ and $8 \mathrm{TeV}$, respectively ${ }^{1}$. Correspondingly, the elastic cross section is $24.00 \pm 0.26$ (stat.) \pm 0.57 (syst.) $\mathrm{mb}$ and $24.33 \pm 0.04$ (stat.) \pm 0.39 (syst.) mb.

In the direct measurement of the inelastic cross section $[9,10]$ the MBTS detector plays a crucial role. It is assumed that an inelastic $p p$ collision occurs when at least two charged particles hitting this detector are observed. The requirement of two hits in MBTS not only removes all elastic $p p$ collisions, but also ensures that in selected events at least one proton dissociates into a system with a mass $M_{X}>13 \mathrm{GeV}$ (at $\sqrt{s}=13 \mathrm{TeV}$ ) or $M_{X}>15.7 \mathrm{GeV}$ (at $\sqrt{s}=7 \mathrm{TeV}$ ). When translated to the variable $\xi=M_{X}^{2} / s$ this corresponds to the fiducial region $\xi>10^{-6}$ and $\xi>5 \times 10^{-5}$ for $13 \mathrm{TeV}$ and $7 \mathrm{TeV}$, respectively. In such kinematical limits the inelastic cross section is $69.1 \pm 2.4$ (exp.) \pm 6.9 (extr.) $\mathrm{mb}$ [9] and $78.1 \pm 0.6$ (exp.) \pm 1.3 (lum.) \pm 2.6 (extr.) $\mathrm{mb}[10]$ at $\sqrt{s}=7 \mathrm{TeV}$ and $\sqrt{s}=13 \mathrm{TeV}$, respectively ${ }^{2}$. These measurements provide additional data for the dependence of the inelastic $p p$ cross section on energy shown in Figure 1, and confirm the trend predicted by Monte Carlo models.

\footnotetext{
${ }^{1}$ Systematic errors are divided into parts related to experimental effects and extrapolation uncertainty.

${ }^{2}$ Systematic errors are divided into parts related to experimental effects, luminosity uncertainty (at $13 \mathrm{TeV}$ ) and extrapolation uncertainty.
}

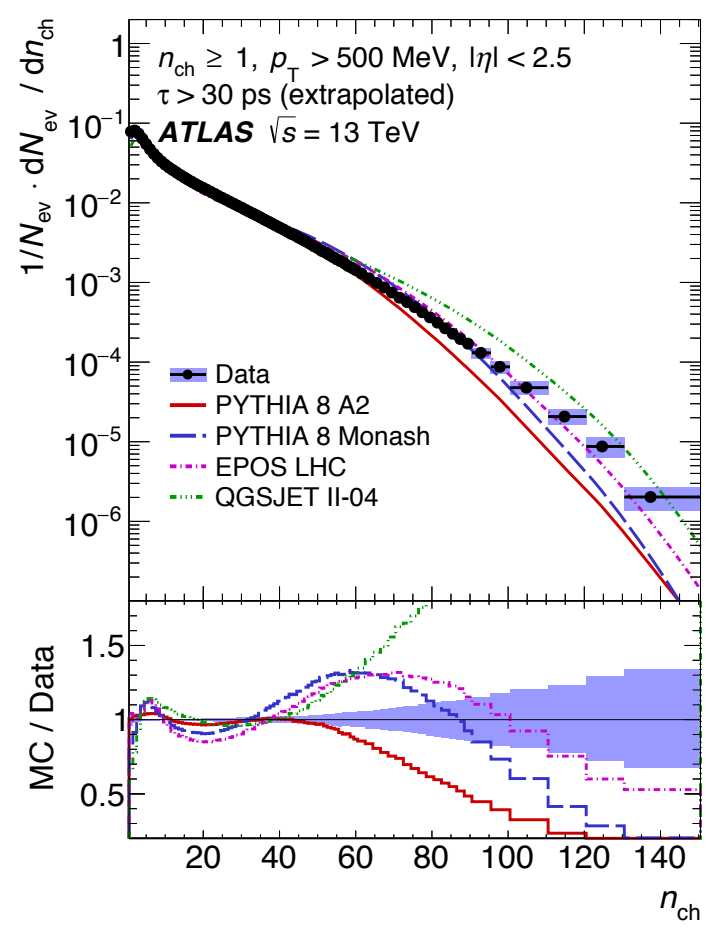

Figure 2. Charged particle multiplicity in $p p$ collisions at $\sqrt{s}=13 \mathrm{TeV}$ [11]. The bottom panel shows the ratio of the MC simulation over the data.

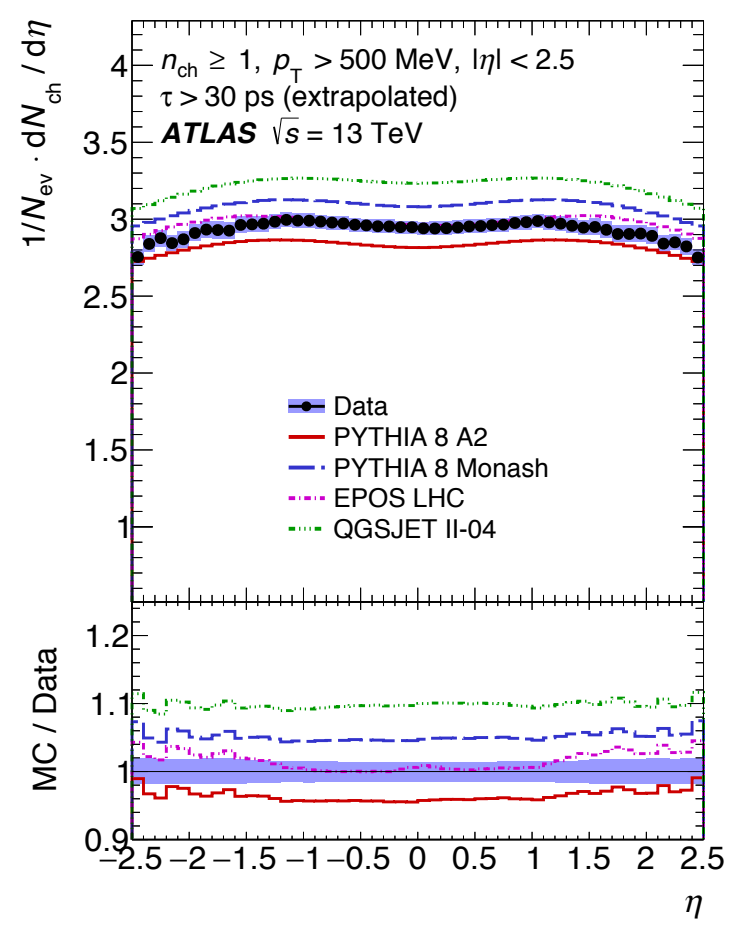

Figure 3. Charged particle pseudorapidity density in $p p$ collisions at $\sqrt{s}=13 \mathrm{TeV}$ [11]. The bottom panel shows the ratio of the MC simulation over the data.

\section{Charged particle production in $p p$ collisions}

Global characteristics of minimum-bias $p p$ events are studied in the ATLAS experiment at all collision ener- 


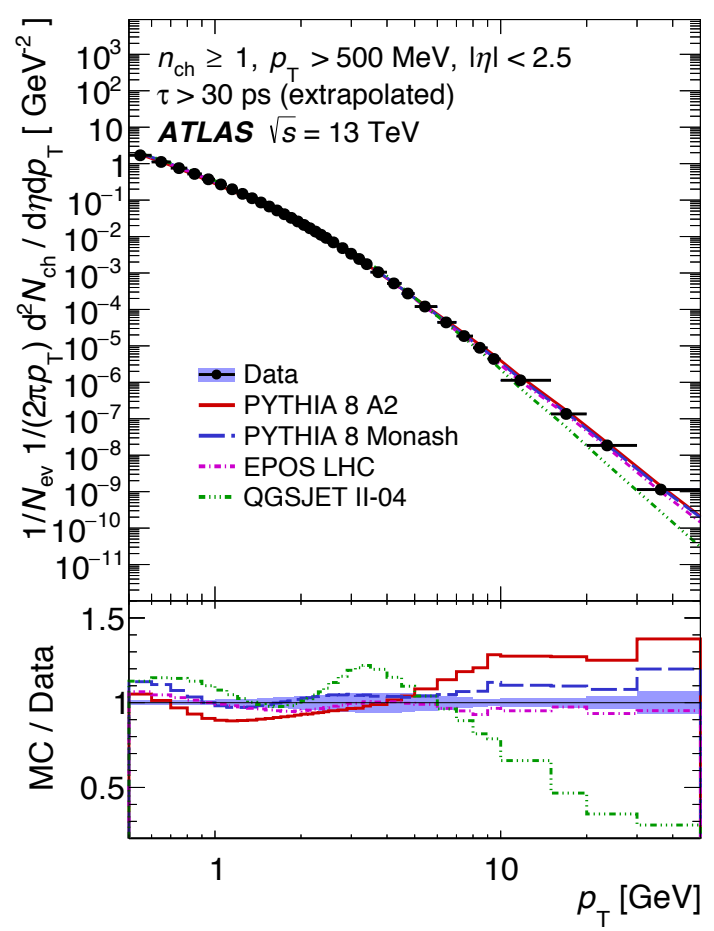

Figure 4. Charged particle transverse momentum distribution in $p p$ collisions at $\sqrt{s}=13 \mathrm{TeV}$ [11]. The bottom panel shows the ratio of the MC simulation over the data.

gies available at the LHC. In the most recent studies at $\sqrt{s}=13 \mathrm{TeV}[11,12]$ distributions of charged particle multiplicity (Figure 2), pseudorapidity (Figure 3) and transverse momentum, $p_{\mathrm{T}}$, (Figure 4) were measured. In the comparison to predictions of several Monte Carlo generators a reasonable agreement is observed. The largest differences can be seen in the multiplicity distribution. They are most significant at low multiplicities, where the contribution from diffraction is present. Best agreement is reached for pseudorapidity distribution: the differences do not exceed $10 \%$. For the $p_{\mathrm{T}}$ distribution, differences with respect to some Monte Carlo models are larger than $20 \%$, but only for $p_{\mathrm{T}}>10 \mathrm{GeV}$. Generally, the EPOS model [13] describes the data most accurately.

The processes most extensively studied by ATLAS require a presence of hard scattered partons. In $p p$ collisions such processes are usually accompanied by additional particle production, the underlying event. The properties of underlying events can be studied by selecting a sample of events with a charged particle with high- $p_{\mathrm{T}}$, the leading particle. The multiplicity of particles from the underlying event depends on their position in azimuth with respect to the leading particle, $\Delta \phi$. In Figure 5 the $\Delta \phi$ distributions for events with a leading particle with $p_{\mathrm{T}}^{\text {lead }}>1 \mathrm{GeV}$ or with $p_{\mathrm{T}}^{\text {lead }}>10 \mathrm{GeV}$ are shown [14]. In the case of more inclusive event sample ( $p_{\mathrm{T}}^{\text {lead }}>1 \mathrm{GeV}$ ) the $\Delta \phi$ distribution is almost flat, but if the $p_{\mathrm{T}}^{\text {lead }}>10 \mathrm{GeV}$ requirement is applied a strong enhancement at $\Delta \phi \approx 0^{\circ}$ and a lesser one at opposite direction, $\Delta \phi \approx 180^{\circ}$, appears.

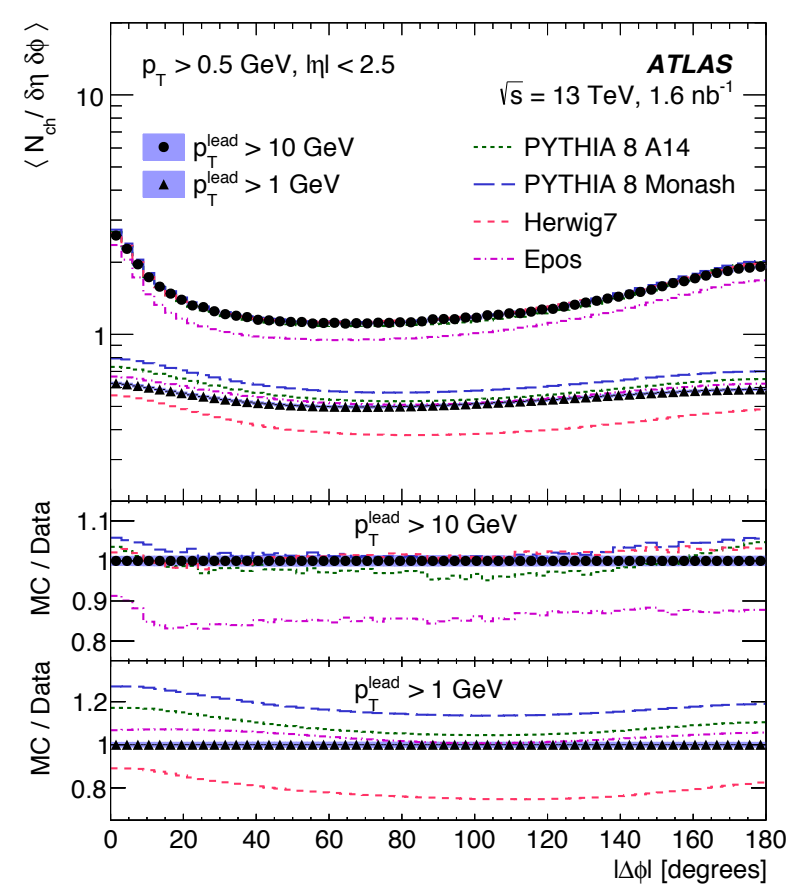

Figure 5. Distribution of the azimuthal angle of charged particles from the underlying event with respect to to the leading charged particle in $p p$ collisions at $\sqrt{s}=13 \mathrm{TeV}$ [14]. The bottom panels show the ratio of the MC simulation over the data.

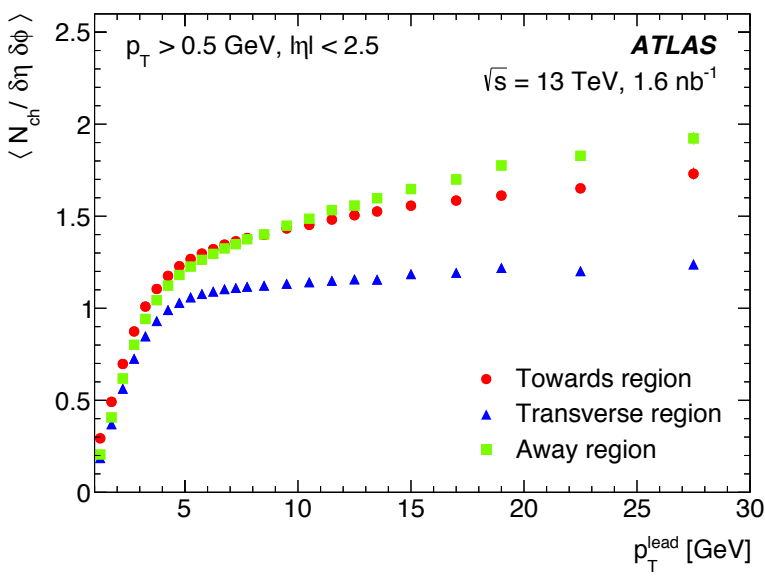

Figure 6. Mean multiplicity density of charged particles from the underlying event in the towards, transverse and away regions as a function of the transverse momentum of the leading charged particle in $p p$ collisions at $\sqrt{s}=13 \mathrm{TeV}$ [14].

Dependences of multiplicity in the towards region $\left(|\Delta \phi|<60^{\circ}\right)$, in the transverse region $\left(60^{\circ}<|\Delta \phi|<120^{\circ}\right)$ and in the away region $\left(|\Delta \phi|>120^{\circ}\right)$ on the $p_{\mathrm{T}}$ of the leading particle are shown in Figure 6 . The multiplicity strongly grows up to $p_{\mathrm{T}}^{\text {lead }} \approx 4 \mathrm{GeV}$ and then the increasing trend gradually flattens, especially for the transverse region. In the events with a high- $p_{\mathrm{T}}$ leading particle the multiplicity in the away region becomes slightly larger than in the towards region, while in the transverse region it is always the smallest. On the other hand the sum of $p_{\mathrm{T}}$ of particles from 


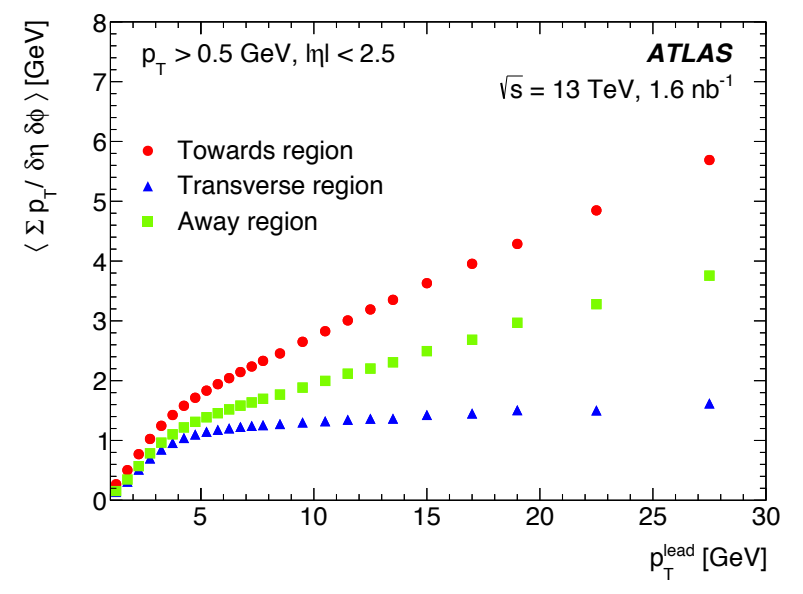

Figure 7. Mean sum of the transverse momenta of charged particles from the underlying event in the towards, transverse and away regions as a function of the transverse momentum of the leading charged particle in $p p$ collisions at $\sqrt{s}=13 \mathrm{TeV}$ [14].

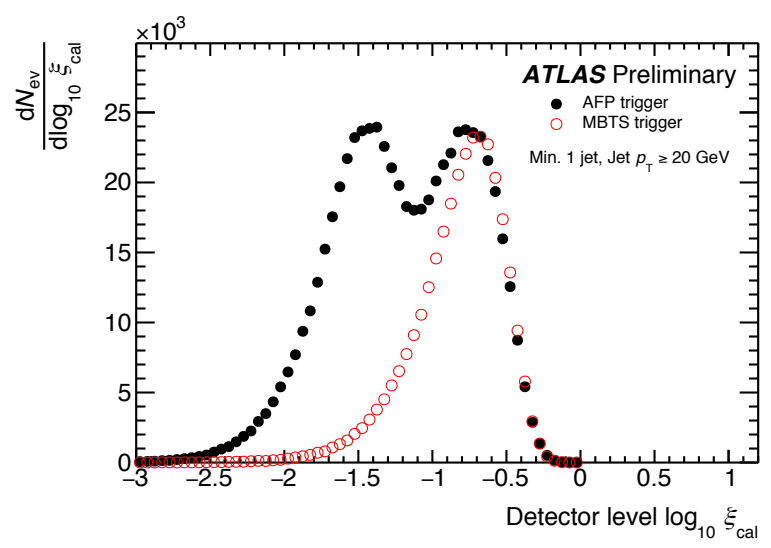

Figure 8. Measured $\xi_{\text {cal }}$ distribution in data samples selected by AFP trigger or by minimum-bias MBTS trigger for events of $p p$ collisions at $\sqrt{s}=13 \mathrm{TeV}$ with at least one jet with $p_{\mathrm{T}}>20 \mathrm{GeV}$ and $|\eta|<3.0$ [5].

the underlying event, shown in Fig. 7, is always the largest in the towards region and the smallest in the transverse region. Above $p_{\mathrm{T}}^{\text {lead }} \approx 4 \mathrm{GeV}$ the slopes of the increasing trend reduce significantly, especially in the transverse region.

\section{Measurements in forward detectors}

In addition to cross-section measurements described in Section 3, the detectors measuring particles emitted at small angles provide information in this kinematic region which is important for proper simulation of the cascades from cosmic rays in the Monte Carlo models.

The LHCf detector is used to measure spectra of very forward photons [15]. Recently these measurements were extended by combining information from LHCf and ATLAS detectors and the inclusive spectra are compared to the spectra for diffractive events, without charged particles in the ATLAS acceptance [16]. More details on results

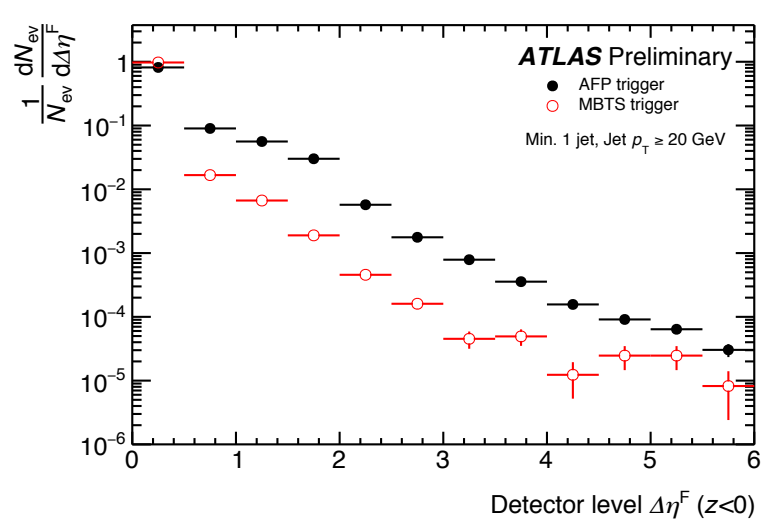

Figure 9. Detector level distributions of the forward rapidity gap, $\Delta \eta^{\mathrm{F}}$, calculated for the negative $z$ (and $\eta$ ) side, in $p p$ collisions at $\sqrt{s}=13 \mathrm{TeV}$ for the AFP and MB trigger data sets [5].

of the LHCf experiment are provided in Takashi Sako's contribution to these proceedings.

The AFP detector is designed for measurements of hard diffractive processes, identified not by the standard rapidity gap method, but thanks to the direct measurement of the forward-scattered proton. The acceptance of the AFP detector allows to register protons for which the fraction of energy loss, $\xi_{\text {cal }}$, is in the range $0.02<\xi_{\text {cal }}<0.1$. This observable is estimated from the energy deposits in all calorimeter cells of the ATLAS detector:

$$
\xi_{\mathrm{cal}}=\frac{1}{\sqrt{s}} \sum_{i} p_{\mathrm{T}}^{i} e^{-\eta_{i}},
$$

where all calorimeter clusters with $p_{\mathrm{T}}>200 \mathrm{MeV}$ are included. Most recently, events of $p p$ collisions at $\sqrt{s}=13 \mathrm{TeV}$ with a jet with $p_{\mathrm{T}}>20 \mathrm{GeV}$ and $|\eta|<3.0$ were analyzed [5]. In Figure 8 the distribution of $\xi_{\text {cal }}$ for events with a proton found in the AFP to that for inclusive inelastic $p p$ collisions (triggered by MBTS) are compared. In both cases at least one jet was also required. The peak at larger $\xi_{\text {cal }}$ values corresponds to non-diffractive collisions, while that at smaller $\xi_{\text {cal }}$ is dominated by diffractive processes. The presence of diffractive processes in events triggered by AFP is confirmed by the rapidity gap analysis shown in Figure 9. The distribution of the detector level pseudorapidity gap, $\Delta \eta^{\mathrm{F}}(z<0)$, a distance between the edge of the calorimeter acceptance $(\eta \approx-4.9)$ and the first calorimeter cell with a signal corresponding to $p_{\mathrm{T}}>200 \mathrm{MeV}$ or a reconstructed charged particle with $p_{\mathrm{T}}>200 \mathrm{MeV}$, shows a much more prominent tail at large $\Delta \eta^{\mathrm{F}}(z<0)$ values for the AFP triggered events than that for MBTS triggered (i.e. inclusive) events.

\section{Heavy-ion collisions}

In interactions involving nuclei the particle production depends mainly on geometrical properties of the collision, i.e. the distance between the centers of the colliding nuclei at the moment of their closest approach - called the impact parameter, $b$. In the case of $\mathrm{Pb}+\mathrm{Pb}$ collisions it determines 


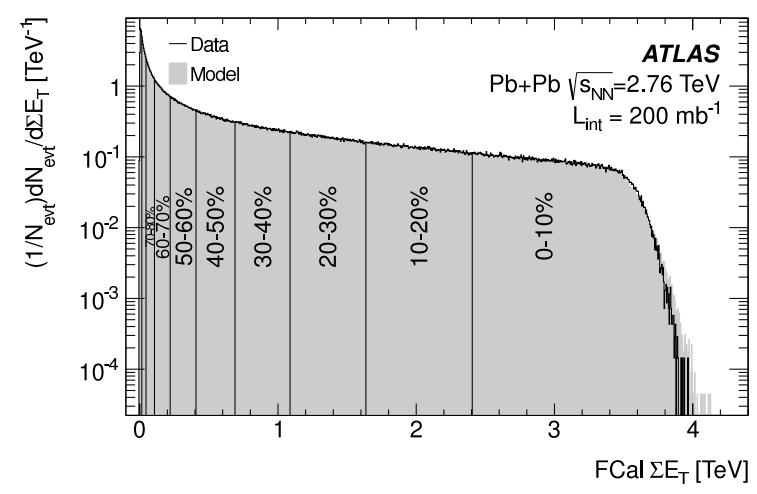

Figure 10. Centrality determination in $\mathrm{Pb}+\mathrm{Pb}$ collisions at $\sqrt{s_{\mathrm{NN}}}=2.76 \mathrm{TeV}[18]$.

the degree of the overlap of nuclei and thus the number of nucleons, which may directly participate in the collision. For $p+\mathrm{Pb}$ collisions the value of the impact parameter decides if the proton hits the nucleus near the center $(b \approx 0)$, where it encounters maximal number of nucleons from lead nuclei, or closer to the edge, where the proton may interact directly with much fewer nucleons. The geometry of the collision may thus be described in terms of the impact parameter, the number of nucleons participating in the collisions, $N_{\text {part }}$, and the number of binary collisions between nucleons, $N_{\text {coll }}$. Relations between $b, N_{\text {part }}$ and $N_{\text {coll }}$ can be obtained from the Glauber model [17]. None of these parameters can be directly measured, but it is possible to find an observable, which is a monotonic function of them. In the ATLAS experiment it is the transverse energy, $\sum E_{\mathrm{T}}$, registered in forward calorimeters, FCal, covering the range $3.2<|\eta|<4$.9. In Figure 10 the distribution of FCal $\sum E_{\mathrm{T}}$ is divided into bins with appropriate percentiles of events, which define the centrality classes, with the most central collisions in the interval 0-10\% [18].

The dependence of particle production in $\mathrm{Pb}+\mathrm{Pb}$ collisions on centrality is best represented in Figure 11, where the charged particle pseudorapidity density is shown in 8 centrality intervals [19]. In the most central events, from the $0-10 \%$ interval, almost 40 times more particles are produced than in peripheral events from the $70-80 \%$ interval.

Interesting results are also provided from the analysis of the energy dependence of the particle density at $\eta \approx 0$ normalized by the number of pairs of nucleons participating in the collision, $N_{\text {part }} / 2$, shown in Figure 12. This normalization allows a direct comparison of nucleus-nucleus collisions and $p p$ collisions, for which $N_{\text {part }}=2$. Just like the mean multiplicity, the $\mathrm{d} N_{\mathrm{ch}} / \mathrm{d} \eta /\left(<N_{\text {part }} / 2>\right)$ is increasing with $\sqrt{s_{\mathrm{NN}}}$, but its values for central collisions of nuclei are about 2 times higher than for $p p$ collisions. This may be explained by the fact that in central collisions nucleons from one nucleus can collide with several nucleons from another nucleus and additional particle production may be due to hard processes which are proportional to the number of nucleon-nucleon collisions, $N_{\text {coll }}$. This picture is supported by the observation that the multiplicity

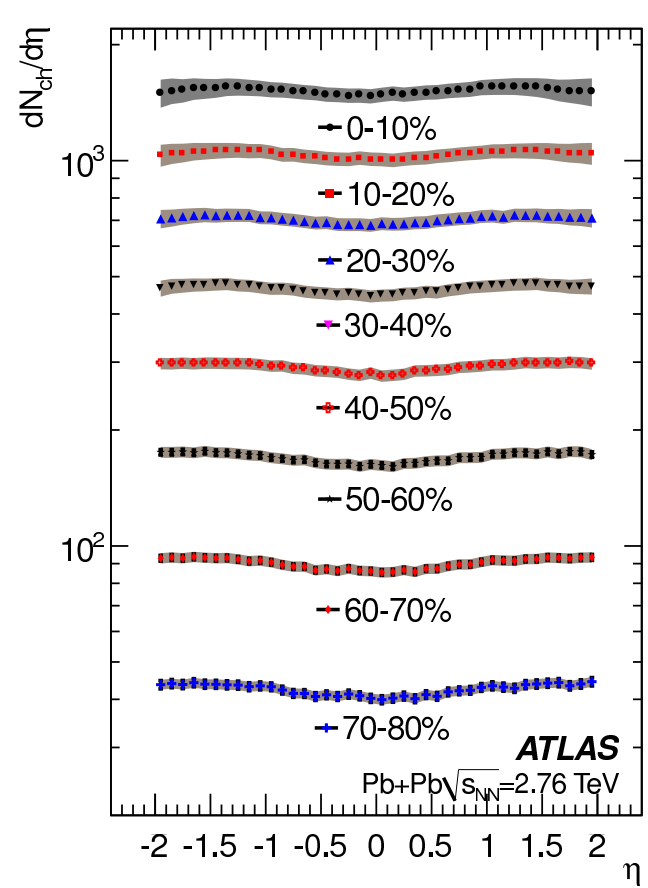

Figure 11. Charged particle pseudorapidity density in $\mathrm{Pb}+\mathrm{Pb}$ collisions at $\sqrt{s_{\mathrm{NN}}}=2.76 \mathrm{TeV}$ for eight centrality intervals [19].

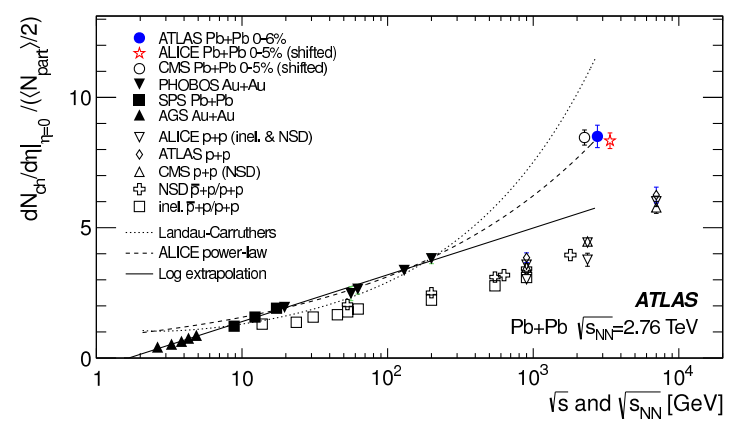

Figure 12. Particle density at $\eta \approx 0$ per pair of nucleons participating in nucleus-nucleus, $p p$ and $p \bar{p}$ collisions as a function of the collision energy [19]

normalized by $N_{\text {part }} / 2$ is a monotonically increasing function of $N_{\text {part }}$, as shown in Figure 13.

A similar analysis was also performed for $p+\mathrm{Pb}$ collisions at $\sqrt{S_{\mathrm{NN}}}=5.02 \mathrm{TeV}[20,21]$. Due to the asymmetry of the colliding system the centrality definition is based on FCal $\sum E_{\mathrm{T}}$, registered in the Pb-going direction only (Fig. 14). This asymmetry is also reflected in the $\mathrm{d} N_{\mathrm{ch}} / \mathrm{d} \eta$ distribution shown in Figure 15. In the most central collisions about $50 \%$ more particles are emitted in the $\mathrm{Pb}$ going direction than in the proton-going direction, while in peripheral collisions the $\mathrm{d} N_{\mathrm{ch}} / \mathrm{d} \eta$ distribution is almost symmetric. The latter is a clear indication that in the very peripheral $p+\mathrm{Pb}$ collisions the proton interacts with one nucleon only.

The multiplicity normalized by $N_{\text {part }} / 2$ presented in Figure 16 as a function of $N_{\text {part }}$ indicates the importance 


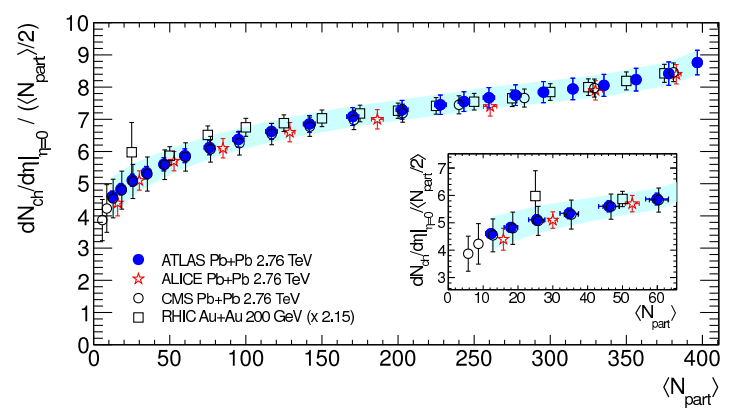

Figure 13. Particle density at $\eta \approx 0$ per a pair of nucleons participating in nucleus-nucleus collisions as a function of the number of nucleons participating in the collision, $N_{\text {part }}$ [19] .

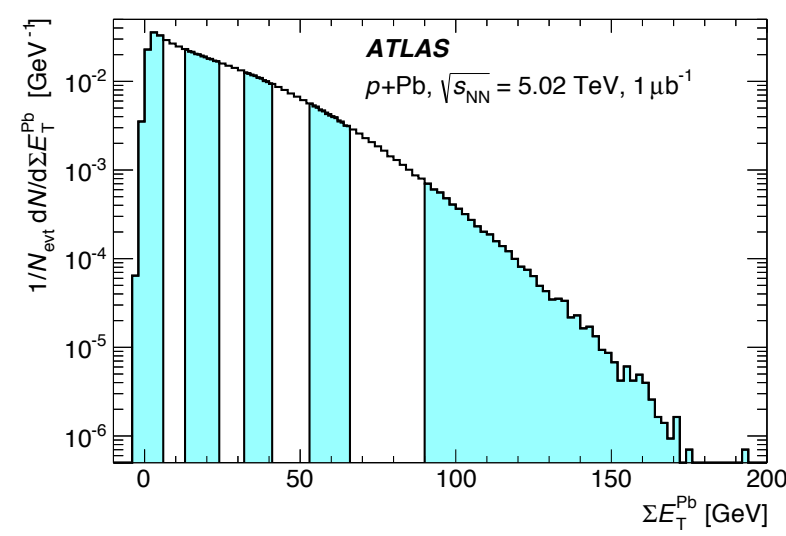

Figure 14. Centrality detemination in $p+\mathrm{Pb}$ collisions at $\sqrt{s_{\mathrm{NN}}}=5.02 \mathrm{TeV}[20]$. The alternating shaded and unshaded bands indicate centrality intervals, from right (central) to left (peripheral), 0-1\%, 1-5\%, 5-10\%, 10-20\%, 20-30\%, 30-40\%, 40-60\%, 60-90\% and 90-100\%

of the proper calculations in the framework of the Glauber model, as the three versions of it (discussed in detail in [20]) give significantly different results, from clearly increasing to slightly decreasing dependence.

In ultra-peripheral collisions of heavy-ions, in which the distance between nuclei is larger than twice their radius, only interactions of their electromagnetic fields occur. These fields, proportional to the charge of the nuclei, $Z$, are additionally enhanced by relativistic effects. In such conditions observation of photon-photon scattering:

$$
\mathrm{Pb}+\mathrm{Pb}(\gamma \gamma) \rightarrow \mathrm{Pb}^{(*)}+\mathrm{Pb}^{(*)} \gamma \gamma
$$

is possible. The measured fiducial cross section of such a process in $\mathrm{Pb}+\mathrm{Pb}$ collisions at $\sqrt{s_{\mathrm{NN}}}=5.02 \mathrm{TeV}$ is $70 \pm 24$ (stat.) \pm 17 (syst.) nb [22] and agrees with theoretical predictions.

\section{Summary}

The Large Hadron Collider provides the highest energy $p p$ and nucleus-nucleus collisions, which are used to search for both new phenomena and to test existing models of par-

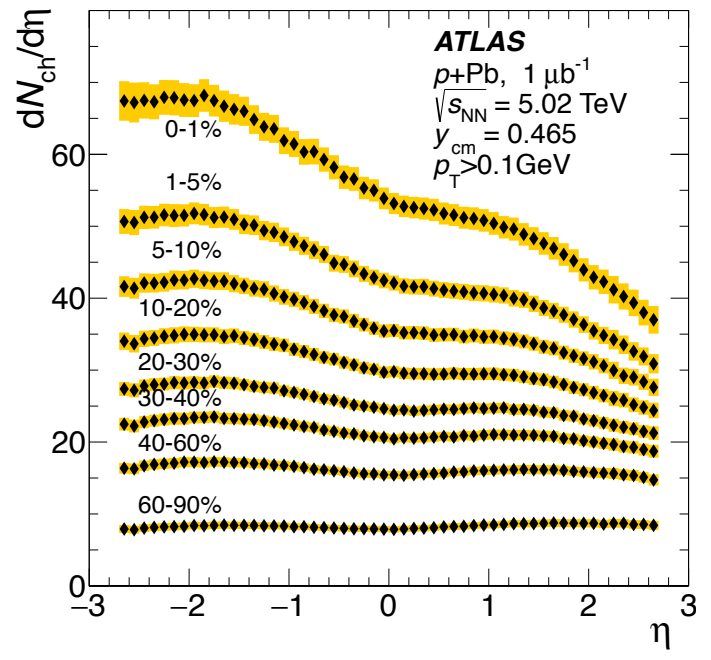

Figure 15. Charged particle pseudorapidity density in $p+\mathrm{Pb}$ collisions at $\sqrt{s_{\mathrm{NN}}}=5.02 \mathrm{TeV}$ for several centrality intervals [20].

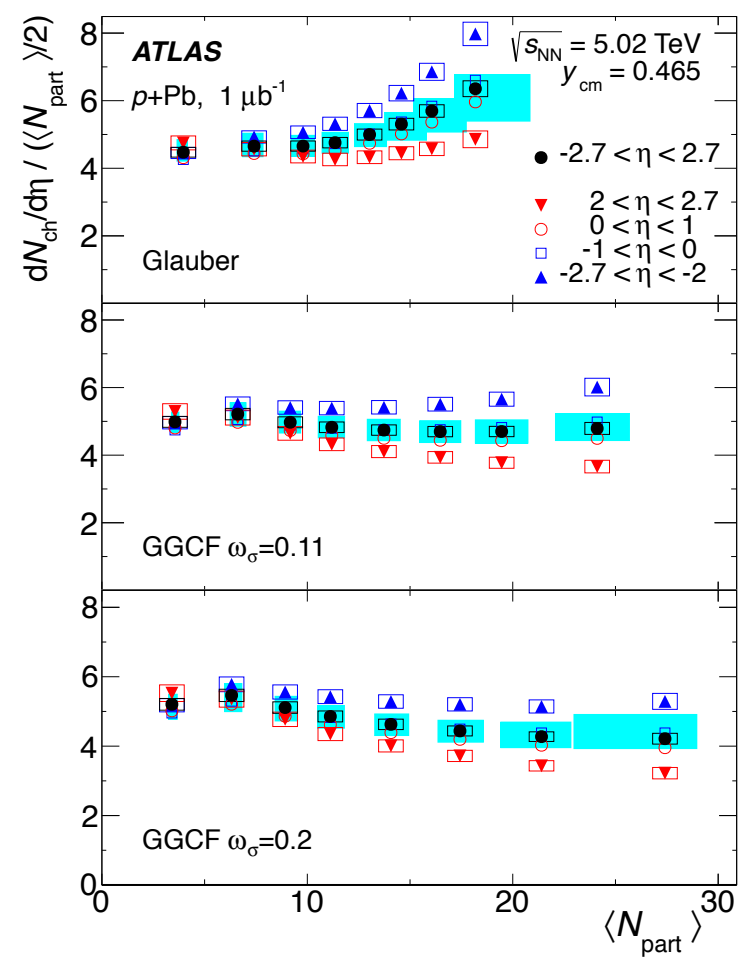

Figure 16. Charged particle pseudorapidity density in $p+\mathrm{Pb}$ collisions at $\sqrt{s_{\mathrm{NN}}}=5.02 \mathrm{TeV}$ as a function of the number of nucleons participating in the collision, $N_{\text {part }}$ [20]. Each panel corresponds to the different version of the Glauber model used to calculate $N_{\text {part }}$.

ticle production. Especially the model testing is important for cosmic ray studies in which primary particles are observed by measurements of showers of particles from interactions in the atmosphere. Realistic values of the cross sections and reproduction of details of particle production are necessary to identify the incoming primary particle and estimate its energy. Although the most frequent cosmic ray interactions in the atmosphere, $p+\mathrm{N}$ or $p+\mathrm{O}$, or inter- 
actions of Fe ions, the heaviest nucleus observed in cosmic rays, were not yet measured at the LHC, the measurements of $p p, p+\mathrm{Pb}$ and $\mathrm{Pb}+\mathrm{Pb}$ collisions can be used to obtain required estimates. The ATLAS experiment delivers a variety of results, which can be helpful in tuning models intended to describe interactions of cosmic ray particles in the $\mathrm{PeV}$ and $\mathrm{EeV}$ energy regions.

Copyright 2018 CERN for the benefit of the ATLAS Collaboration. Reproduction of this article or parts of it is allowed as specified in the CC-BY-4.0 license.

\section{Acknowledgements}

This work was supported in part by the National Science Centre, Poland grant 2016/23/B/ST2/00702 and by PL-Grid Infrastructure.

\section{References}

[1] ATLAS Collaboration, Phys. Lett. B 716, 1 (2012)

[2] CMS Collaboration, Phys. Lett. B 716, 30 (2012)

[3] ATLAS Collaboration, JINST 3, S08003 (2008)

[4] S.A. Khalek, B. Allongue, F. Anghinolfi, P. Barrillon, G. Blanchot, S. Blin-Bondil, A. Braem, L. Chytka, P.C. Muíño, M. Düren et al., JINST 11, P11013 (2016)

[5] ATLAS Collaboration, ATL-PHYS-PUB-2017-012 (2017), https://cds . cern . ch/record/2265187
[6] O. Adriani, L. Bonechi, M. Bongi, G. Castellini, R. D’Alessandro, D. Faus, K. Fukui, M. Grandi, M. Haguenauer, Y. Itow et al., JINST 3, S08006 (2008)

[7] ATLAS Collaboration, Nucl. Phys. B 889, 486 (2014)

[8] ATLAS Collaboration, Phys. Lett. B 761, 158 (2016)

[9] ATLAS Collaboration, Nature Commun. 2, 463 (2011)

[10] ATLAS Collaboration, Phys. Rev. Lett. 117, 182002 (2016)

[11] ATLAS Collaboration, Phys. Lett. B 758, 67 (2016)

[12] ATLAS Collaboration, Eur. Phys. J. C 76, 502 (2016)

[13] S. Porteboeuf, T. Pierog, K. Werner, arXiv:1006.2967 [hep-ph] (2010)

[14] ATLAS Collaboration, JHEP 03, 157 (2017)

[15] O. Adriani, E. Berti, L. Bonechi, M. Bongi, R. D’Alessandro, M. Haguenauer, Y. Itow, T. Iwata, K. Kasahara, Y. Makino et al., arXiv:1703.07678v2 [hep-ex] (2017)

[16] ATLAS Collaboration, ATLAS-CONF-2017-075 (2017), https://cds.cern.ch/record/2291387

[17] M. Miller, K. Reygers, S. Sanders, P. Steinberg, Rev. Nucl. Part. Sci. 57, 205 (2007)

[18] ATLAS Collaboration, Phys. Lett. B 707, 330 (2012)

[19] ATLAS Collaboration, Phys. Lett. B 710, 363 (2012)

[20] ATLAS Collaboration, Eur. Phys. J. C 76, 199 (2016)

[21] ATLAS Collaboration, Phys. Lett. B 763, 313 (2016)

[22] ATLAS Collaboration, Nature Phys. p. 852 (2017) 